\title{
Caracterización espectroscópica y cromatográfica de curcumina extraída de los rizomas de Cúrcuma (cúrcuma longa l.) Cultivada en el departamento del Quindío
}

\section{Spectroscopy and chromatography characterization of curcumin extracted from the rhizome of turmeric crops in the department of Quindío (Colombia)}

\author{
Eunice Ríos V.* Alba Lucia Duque C. **, David Fabián León R.***
}

Recibido: agosto 10 de 2009

Aceptado: octubre 22 de 2009

Correspondencia: Maestria en Quìmica, Universidad del Quindio, Avenida Bolivarcalle 12 norte, Armenia Quindìo. Email:erios@uniquindio.edu.co

\section{RESUMEN}

La extracción de compuestos de origen natural es una de las industrias en mayor crecimiento debido a los beneficios que presentan frente a sus análogos de origen sintético, en esta investigación se extrajo el principal compuesto coloreado de los rizomas de la cúrcuma (Curcuma longa L.): Curcumina con rendimiento del 7.03\% tras 9 horas de extracción, utilizando soxhlet con solventes orgánicos (hexano y etanol). La separación y purificación se realizó por cromatografía de capa delgada y de columna, usando como fase móvil una mezcla de cloroformo y acetato de etilo. El colorante fue caracterizado por análisis espectroscópicos de ultravioleta visible (UV-Vis), infrarrojo (IR), resonancia magnética nuclear (1D-RMN y 2D-RMN) y espectroscopia de masas (EM). Los resultados obtenidos de los análisis fisicoquímicos de humedad, cenizas, extracto etéreo, contenido de fibras y contenido de curcumina de los rizomas de la región fueron comparados con los reportes de otras regiones del mundo, encontrando notables diferencias entre los parámetros comparados.

Palabras Clave: Curcuma longa Linn, turmeric, curcumina, extracción soxhlet, cromatografía.

\begin{abstract}
The extraction of natural compounds is one of the fastest growing industries due to the benefits they have compared to their synthetic analogs. In this investigation, the curcumin was extracted from the rhizome of turmeric (Curcuma longa L.) with a yield of $7.03 \%$ after 9 hours of extraction using soxhlet extractors with organic solvents (hexane and ethanol). The separation and purification was performed by thin layer and column chromatography using a mixture of chloroform and ethyl acetate as mobile phase. The curcumin was characterized by spectroscopic analysis of ultraviolet visible (UV-Vis), infrared (IR), nuclear magnetic resonance (NMR-1D and 2D-NMR) and mass spectroscopy (MS). The results of the physicochemical analysis of moisture, ashes, ether extract, fiber content and curcumin content from the rhizomes in the region were compared with reports from other regions of the world, finding significant differences between the parameters compared.
\end{abstract}

Keywords: Curcuma longa Linn, turmeric, curcumin, soxhlet extraction, chromatography

\section{INTRODUCCIÓN}

os suplementos son adicionados a los alimentos con el fin de mejorar su apariencia, sabor, color, y ayudar a su preservación. Actualmente hay un considerable interés mundial en el desarrollo de los colorantes naturales debido a su seguridad y beneficio para el organismo. (1)
La cúrcuma (Cúrcuma longa Linn) conocida también como turmeric o haldi (Asia), de origen asiático pertenece a la familia Zingiberaceae. Se cultiva principalmente en China, India, Indonesia, Jamaica y Perú. Las propiedades del turmeric son muy importantes para la industria debido a que cuando se adiciona a preparaciones alimentarias preserva su frescura e imparte un sabor característico además, de

\footnotetext{
*erios@uniquindio.edu.co. Grupo de Investigación Agroindustria de Frutas Tropicales, Programa de Química, Universidad del Quindío. **Albduque@uniquindio.edu.co, Grupo de Investigación Agroindustria de Frutas Tropicales, Programa de Química, Universidad del Quindío.

***davidfabianleon@hotmail.com, Grupo de Investigación Agroindustria de Frutas Tropicales, Programa de Química, Universidad del Quindío.
} 
su uso en la medicina tradicional principalmente contra afecciones estomacales. (2)

En la medicina ayurvédica (que en la India significa "ciencia de la vida"), la curcumina es utilizada para diversas afecciones respiratorias (por ejemplo, el asma, hiperactividad bronquial y alergia), así como para trastornos hepáticos, anorexia, el reumatismo, la diabetes, nariz con mucosidad, tos y sinusitis. En la medicina tradicional china, se usa para el tratamiento de enfermedades asociadas con dolor abdominal. En la antigua medicina hindú, es utilizada para el tratamiento de esguinces y la hinchazón. En todo el Oriente, tradicionalmente ha sido utilizada como buen tratamiento terapéutico, en particular como anti-inflamatorio, y muchos de sus efectos terapéuticos han sido confirmados por la investigación científica moderna. Estos efectos incluyen antioxidante, antiinflamatorios, anticancerígeno y antimicrobianos, hepatoprotector, trombosupresivo, cardiovascular (protección contra el infarto de miocardio), hipoglucemiantes y protección contra la artritis reumatoide. (3).

La cúrcuma cuenta en su composición química con compuestos volátiles y no volátiles. Los principales compuestos volátiles encontrados son: cariofileno, arcurmeno, zingibereno, bisaboleno, sesquifelandrenendreno (4), y tres turmeronas (5); dentro de los compuestos no volátiles más sobresalientes se encuentra un péptido soluble en agua llamado turmerina (6) y polifenoles (7).

La curcumina es el principal polifenol curcuminoide encontrado en el turmeric, junto con la demetoxicurcumina, bisdemetoxicurcumina y la recientemente descubierta ciclocurcumina forman el complejo conocido como azafrán indio, raíz amarilla, jengibre amarillo o amarillo natural 3. La curcumina $\left(\mathrm{C}_{21} \mathrm{H}_{20} \mathrm{O}_{6}\right)$ es también conocida como diferuloilmetano o 1,7-bis-(4-hidroxi-3-metoxifenil)-1,6heptadieno-3,5-diona, es un compuesto enólico de bajo peso molecular $(369.37 \mathrm{~g} / \mathrm{mol})$ con punto de fusión 183 으, de color amarillo en medio ácido ( $\mathrm{pH} 2,5-7)$ y rojo en medio básico $(\mathrm{pH}$ $>7$ ), es soluble en solventes orgánicos como dimetilsulfoxido, etanol, metanol o acetona y muy poco soluble en solventes acuosos. (8)

Ha sido extraído y separado por diversos métodos como: soxhlet, maceración-agitación, extracción asistida por microondas (MWHD) (9), cromatografía de capa delgada y columna (10) y cromatografía líquida de alta resolución. (11)

El objetivo de esta investigación fue extraer, cuantificar y caracterizar espectroscópica y cromatográficamente la curcumina de los rizomas de cúrcuma
(Cúrcuma longa L.) cultivada en el Departamento del Quindío, dándole un valor alto de estudio, el hecho de ser cultivada en esta Región, para su uso ya sea en la industria alimentaria, cosmética o química.

\section{MATERIALES Y MÉTODOS}

\section{Materia Prima:}

Los rizomas de la cúrcuma fueron aportados por la Asociación Bioagroindustrial (ASOBAI), cultivados a 1294 a.s.n.m. en el corregimiento de Pueblo Tapao, Municipio de Montenegro, Departamento del Quindío.

\section{Caracterización Fisicoquímica de los Rizomas Frescos:}

A los rizomas frescos se les realizaron los siguientes análisis, los cuales fueron comparados con rizomas de otras regiones:

Humedad (Método A.O.A.C. 930.15/90 Modificado): se pesó una cantidad constante de muestra en una cápsula de porcelana previamente tarada, se llevó a un horno de aire caliente a $110^{\circ} \mathrm{C}$ por $2 \mathrm{~h}$, transcurrido este tiempo se pesaron las cápsulas y se llevaron al desecador por una hora, se pesaron e introdujeron al horno por $1 / 2$ hora, se pesaron de nuevo las cápsulas y se repitió el procedimiento anterior hasta alcanzar peso constante.

Cenizas (Método A.O.A.C. 942.05/90 Modificado): se pesó una cantidad constante de las muestras previamente secadas a $110^{\circ} \mathrm{C}$, luego fueron carbonizadas al mechero para posteriormente ser llevadas a una mufla a $600^{\circ} \mathrm{C}$ hasta alcanzar el peso constante de las muestras.

Actividad de Agua ( $\boldsymbol{a}_{w}$ ): se determinó con un higrómetro de punto de rocío (Aqualab Decagon modelo 3TE) de cuatro cifras significativas, realizando por triplicado el análisis de cada muestra.

Extracto etéreo: Se determinó por el método Soxhlet utilizando un equipo P-Selecta usando éter etílico como disolvente.

Contenido de Fibras (Método A.O.A.C. 985.29/90 Modificado): Se peso una cantidad constante del material vegetal desengrasado y se sometió a hidrólisis con $200 \mathrm{~mL}$ de $\mathrm{H} 2 \mathrm{SO} 4$ al $1.25 \%$ por 30 minutos, el residuo se filtró y lavó con agua hasta $\mathrm{pH}$ neutro, luego se sometió nuevamente a hidrólisis por 30 minutos con $200 \mathrm{~mL}$ de $\mathrm{NaOH} 1.25 \%$; el residuo filtrado se lavó con agua destilada hasta $\mathrm{pH}$ neutro, luego se secó en un horno a $110^{\circ} \mathrm{C}$ por 2 horas, se pesó el residuo seco y por último se llevó a una mufla a $600^{\circ} \mathrm{C}$ hasta obtener cenizas blancas las cuales fueron pesadas. 


\section{Preparación de Harina:}

Los rizomas fueron cortados en rodajas y secados por circulación con aire caliente a $40^{\circ} \mathrm{C}$ por dos días, se molieron y tamizaron hasta obtener un tamaño de partícula adecuado para las extracciones, la harina obtenida fue almacenada para los análisis posteriores.

\section{Extracción del Colorante:}

La harina fue desengrasada con hexano durante 9 horas. El material desengrasado se sometió a extracción soxhlet con etanol absoluto por 9 horas con el fin de obtener el extracto de colorantes.

\section{Separación y Purificación de Curcumina:}

El extracto se concentró y analizó por cromatografía de capa delgada utilizando placas de sílica gel y diferentes solventes, como fase móvil. La separación del extracto se hizo por columna cromatográfica. Una posterior purificación para la caracterización de esta fue realizada por cromatografía preparativa.

\section{Caracterización Espectroscópica:}

Análisis por Ultravioleta visible (UV-Vis): fue realizado en un espectrofotómetro de ultravioleta visible Hewlett-Packard 8453 , usando etanol y cloroformo como blancos y una celda de cuarzo de $1 \mathrm{~cm}$ de longitud.

Análisis por Infrarrojo (IR): fue realizado en un equipo FTIR Thermo Nicolet 340, usando pastillas de $\mathrm{KBr}$ grado espectrofotometría infrarroja.

Análisis por Resonancia Magnética Nuclear (RMN): se realizaron análisis de $1 \mathrm{D}$ RMN $\left({ }^{3} \mathrm{C},{ }^{1} \mathrm{H}\right.$ ) y $2 \mathrm{D} \mathrm{RMN}$ (COSY, HMBC y HSQC) en un equipo Broker $\mathrm{AMX}$ de $400 \mathrm{MHz}$, usando cloroformo deuterado como disolvente.

Análisis por Espectrometría de Masas: Se realizó por inyección directa de la muestra al detector en un cromatógrafo de gases acoplado a masas (Shimadzu GCMSQP2010) por impacto electrónico (IE) a 70eV.

\section{RESULTADOS Y DISCUSIÓN}

\section{Caracterización de los Rizomas:}

Con los análisis fisicoquímicos realizados se obtuvo la caracterización de los rizomas cultivados en el Departamento de Quindío (Tabla 1), encontrando diferencias con reportes de otra región (13), dando contenidos de agua, materia inorgánica y fibras más altos debido a las características ambientales y fisiológicas de los rizomas, se podría pensar en el hecho, de ser cultivados en una región más húmeda con temperaturas más bajas y una mayor altura sobre el nivel del mar, estos parámetros pueden alteran las composiciones químicas tal como se pudo observar en los rizomas de ambas regiones.

Tabla 1. Parámetros Fisicoquímicos de los Rizomas de Cúrcuma.

\begin{tabular}{|l|c|c|}
\hline \multicolumn{1}{|c|}{ Pará m etro } & $\begin{array}{c}\text { Quindío } \\
\text { (Colom b ia) }\end{array}$ & $\begin{array}{c}\text { Portugues a } \\
\text { (Venezue la) }\end{array}$ \\
\hline $\begin{array}{l}\text { Altura Sobre el Nivel del } \\
\text { Mar (m) }\end{array}$ & 9004750 & 701200 \\
\hline $\begin{array}{l}\text { Temperatura Promedio } \\
\left({ }^{\circ} \mathrm{C}\right)\end{array}$ & 1821 & 2229 \\
\hline Humedad Relativa (\%) & 80 & 70 \\
\hline Humedad $(\%)$ & $84.73 \pm 2.99$ & $72,63 \pm 1,34$ \\
\hline Cenizas (\%) & $3.90 \pm 1.07$ & $1,40 \pm 0,05$ \\
\hline Actividad de Agua & $0.990 \pm 0.002$ & N.R. \\
\hline Extracto Etéreo (\%) & $0.051 \pm 0.02$ & $7,54 \pm 0,29$ \\
\hline Contenido de Fibra (\%) & $11.88 \pm 1.05$ & $8,35 \pm 0,38$ \\
\hline
\end{tabular}

La elevada humedad y actividad de agua de los rizomas los hace más propensos a daños por microorganismos haciendo que su vida útil en estado fresco sea corta (menor de 1 semana), razón por la cual los rizomas frescos luego de recibidos, lavados y desinfectados fueron cortados y secados para obtener la harina, la cual tiene un periodo de vida de anaquel mucho más largo.

\section{Extracción de Curcumina}

Al final del proceso de extracción con etanol se obtuvo un extracto concentrado de color rojo-naranja, se analizó por cromatografía de capa delgada (CCD) utilizando para la separación del compuesto una mezcla de cloroformo y acetato de etilo $(7: 1)$ mostrando tres manchas de color amarillo con $\mathrm{Rf} 0.35,0.40$, y 0.50 , la mancha de mayor $\mathrm{Rf}$ por su carácter no polar correspondió a la curcumina.

\section{Separación Cromatográfica}

Se realizó una separación cromatográfica con cloroformo como fase móvil, mostrando una buena separación de la primera con respecto a la segunda (Rf1: 0.25 y $\mathrm{Rf2}$ : 0.075$)$, lo cual fue confrontado con el blanco del extracto inicial por CCD. Las fracciones recolectadas cristalizaron con un porcentaje de rendimiento del $7.03 \%$ una vez eliminado el solvente, con estos cristales se realizaron los análisis espectroscópicos. Al analizar los contenidos de curcumina en Colombia (7.03\%), con los reportes para Venezuela (3.06 $\%$ y y la India de (4.98\%), se pudo observar que el contenido es mayor en la cúrcuma de la región del Quindío (Colombia) con respecto a los otros países ( 9 y 13), elevando el interés por la investigación de este compuesto para su posible uso en la industria de los alimentos, cosmética y/o química y 
contribuyendo al desarrollo agroindustrial de la región.

\section{Análisis Espectroscópicos:}

Los espectros UV-Vis obtenidos de los cristales presentaron absorciones máximas a $\lambda$ max EtOH nm (Log $\varepsilon$ ): 426 (2.7) y $\lambda \max \mathrm{CHCl}_{3} \mathrm{~nm}(\log \varepsilon): 418$ (2.6), causados por la múltiple conjugación de enlaces en la molécula, estos valores de $\lambda \max$ concuerdan con los datos reportados para la curcumina.

El espectro IR de los cristales mostró diversas bandas características de la curcumina como $\lambda \max \mathrm{KCl} \mathrm{cm}-1$ : d-1629 $(>\mathrm{C}=\mathrm{O})$ debilitada por la múltiple conjugación de enlaces en la estructura; la acción de los metoxilos impide formación de puentes de hidrógeno con los grupos hidroxilo evidenciándose en $\lambda \operatorname{max~} \mathrm{KCl} \mathrm{cm}{ }^{-1}: \mathrm{d}-1629(>0-\mathrm{H})$; y otras bandas como $\lambda \operatorname{max~KCl~cm} 1:$ m-2925.31 (>C-C-H), $\lambda \max \mathrm{Kcl}$ $\mathrm{cm}^{-1}: \mathrm{mf}-1511.45$ (>C=C), $\lambda \operatorname{max~KCl~cm} \mathrm{m}^{-1}: \mathrm{m}-1204.99$ (>C-O) y $\lambda \operatorname{max~KCl~cm}{ }^{-1}: \mathrm{d}-963.90$ (>C $=\mathrm{C}$ trans. Los análisis realizados $\mathrm{y}$ las correlaciones hechas a los espectros de 1D-RMN y 2DRMN se muestran en la tabla 2.

Tabla 2. Análisis por RMN Unidimensional y Bidimensional.

\begin{tabular}{|c|c|c|c|c|c|}
\hline Posición & ${ }^{1} \mathrm{H}-\mathrm{RMN}(\delta)$ & ${ }^{13} \mathrm{C}-\mathrm{RMN}(\delta)$ & cosy & HSQC & $\mathrm{HMBC}$ \\
\hline$C-1$ & $7.6(\mathrm{~d}, 2 \mathrm{H})$ & 140.5 & $\mathrm{H}-2$ & $\mathrm{H}-1$ & H-2" \\
\hline C-2 & $6.5(\mathrm{~d}, 2 \mathrm{H})$ & 121.8 & $\mathrm{H}-1$ & $\mathrm{H}-2$ & $\mathrm{H}-1, \mathrm{H}-2 "$ \\
\hline$C-3$ & & 183.2 & & & $\mathrm{H}-1, \mathrm{H}-2$ \\
\hline \multicolumn{6}{|l|}{ C-4 } \\
\hline $\mathrm{C}-5$ & & 183.2 & & & $\mathrm{H}-6, \mathrm{H}-7$ \\
\hline C-6 & $6.5(\mathrm{~d}, 2 \mathrm{H})$ & 121.8 & $\begin{array}{l}\mathrm{H}-7 \\
\end{array}$ & $\mathrm{H}-6$ & $\mathrm{H}-7, \mathrm{H}-\mathrm{2}^{\prime}$ \\
\hline C-7 & $7.6(\mathrm{~d}, 2 \mathrm{H})$ & 140.5 & $\mathrm{H}-6$ & $\mathrm{H}-7$ & $\mathrm{H}-2^{\prime}$ \\
\hline C-1' & & 127.7 & & & $\mathrm{H}-6, \mathrm{H}-5^{\prime}$ \\
\hline C-2' & $7.1(\mathrm{~s}, 2 \mathrm{H})$ & 109.7 & $\mathrm{H}-5^{\prime}$ & $\mathrm{H}-2^{\prime}$ & $\mathrm{H}-7, \mathrm{H}-6^{\prime}$ \\
\hline C-3' & $\begin{array}{c}\mathrm{OCH}_{3} 3.9 \\
(\mathrm{~s}, 6 \mathrm{H})\end{array}$ & $\left.{ }^{146.8} \mathrm{OCH}_{3} 56.0\right)$ & & $\mathrm{OCH}_{3}$ & $\mathrm{H}-\mathrm{2}^{\prime}, \mathrm{H}^{\mathrm{H}-5^{\prime}, \mathrm{H}-\mathrm{O}^{\prime}}$ \\
\hline C-4' & & 147.9 & & & $\mathrm{H}-2, \mathrm{H}^{-5} \mathrm{OCH}_{3}^{3} \mathrm{H}-6$ \\
\hline C-5' & $6.9(\mathrm{~d}, 2 \mathrm{H})$ & 114.8 & $\mathrm{H}-2^{\prime}$ & H-5' & \\
\hline C-6' & $7.1(\mathrm{~d}, 2 \mathrm{H})$ & 122.8 & & $\mathrm{H}-6^{\prime}$ & $\mathrm{H}-7, \mathrm{H}-2^{\prime}$ \\
\hline C-1" & & 127.7 & & & $\mathrm{H}-2, \mathrm{H}-5^{\prime \prime}$ \\
\hline C-2" & $7.1(\mathrm{~s}, 2 \mathrm{H})$ & 109.7 & $\mathrm{H}-5^{\prime \prime}$ & $\mathrm{H}-\mathrm{2}^{\prime \prime}$ & $\mathrm{H}-1, \mathrm{H}-6 "$ \\
\hline C-3" & $\begin{array}{c}\mathrm{OCH}_{3} 3.9 \\
\mathrm{~s}, 6 \mathrm{H})\end{array}$ & $\begin{array}{ll}146.8 \\
\left(\mathrm{OCH}_{3} 56.0\right)\end{array}$ & & $\mathrm{OCH}_{3}$ & $\begin{array}{l}\mathrm{H}-2^{\prime \prime}, \mathrm{H}-5^{\prime \prime}, \mathrm{H}-\mathrm{C}^{\prime} \\
\mathrm{OCH}_{3}\end{array}$ \\
\hline C-4" & & 147.9 & & & $\begin{array}{c}\mathrm{H}-\mathrm{-2}^{\prime \prime}, \mathrm{H}-\mathrm{-}^{\prime \prime}, \mathrm{H}-\mathrm{-}^{\prime} \\
\mathrm{OCCH}_{3}\end{array}$ \\
\hline C-5" & $6.9(\mathrm{~d}, 2 \mathrm{H})$ & 114.8 & $\mathrm{H}-2^{\prime \prime}$ & H-5" & \\
\hline C-6" & $7.1(\mathrm{~d}, 2 \mathrm{H})$ & 122.8 & & H-6" & $\mathrm{H}-1, \mathrm{H}-2$ " \\
\hline
\end{tabular}

El análisis realizado por espectrometría de masas muestra fragmentos a $368[\mathrm{M}]+(16.5), 350[\mathrm{M}-\mathrm{H} 2 \mathrm{O}]+$ (21.9), 177 [M-191]+ (33.8), $137[\mathrm{M}-231]+(40.5), 97[\mathrm{M}-271]+(75.3)$,
$83[\mathrm{M}-285]+(81.1)$, y $57[\mathrm{M}-311]+(100.0)$ estos picos confirman el peso molecular de la curcumina para la elucidación total de su estructura. (Figura 1).Una aproximación al mecanismo de la fragmentación de la curcumina se muestra en la figura 2 . En esta se pueden observar los iones correspondientes al peso molecular (ion molecular) y al pico base, el mecanismo inicia con la ionización y pérdida de uno de los grupos metoxilos y el rompimiento del anillo bencénico correspondiente, para llegar a la formación del ion acilio y la fragmentación final del anillo bencénico restante.

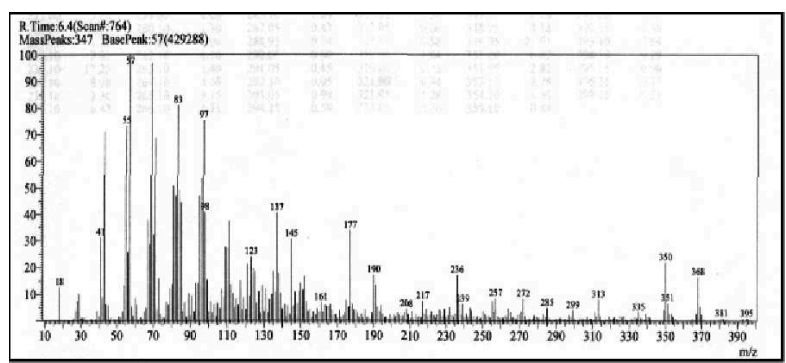

Figura 1. Espectro de masas para la curcumina<smiles>CCOc1cc(/C=C/C(=O)CC(O)/C=C/c2ccc(O)c(OC)c2)ccc1I</smiles><smiles>CCc1cc(/C=C/C(=O)CC(=O)/C=C/c2ccc(CCBr)cc2)ccc1C</smiles><smiles>CCc1cc(/C=C/C(=O)CC(C)/C=C/C2CC[C@H]2C)ccc1Br</smiles><smiles>O=C(C=CC=Cc1ccc(Cl)c(CO)c1)CC(=O)C1CC1</smiles>

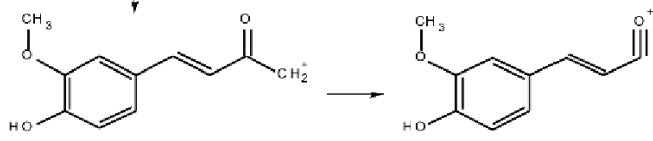

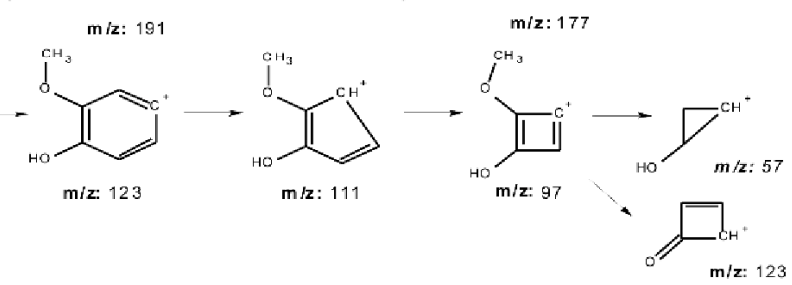

Figura 2. Fragmentación propuesta para la curcumina

\section{AGRADECIMIENTOS}

A la Universidad del Quindío, por el apoyo financiero para esta investigación y A la Asociación Bioagroindustrial ASOBAl, por el aporte del material vegetal. 


\section{BIBLIOGRAFÍA}

(1) Tafoya, A. y García F. Colorantes. En: Biotecnología Alimentaria. México: Limusa. 1993. pp. 479-617.

(2) Jayaprakasha, G.K.; L.J. Rao y K.K. Sakariah. Chemistry and biological activities of C. Longa. Trends in Food Science and Technology. 2005. 16 (12): 533-548.

(3) Goel, A.; A.B. Kunnumakkara y B.B. Aggarwal. Curcumin as 'Curecumin': From kitchen to clinic. Biochemical Pharmacology. 2008. 75 (4): 787-809

(4)Qin, N.Y.; Yang F.Q.; Wang Y.T. y S.P. Li. Quantitative determination of eight components in rhizome (Jianghuang) and tuberous root (Yujin) of Curcuma longa using pressurized liquid extraction and gas chromatography-mass spectrometry. Journal of Pharmaceutical and Biomedical Analysis. 2007. 43 (2): 486-492.

(5) Rios V. E.; Giraldo G.G.A.; León R.D.F.; Moreno B.A.; Estudio del perfil volátil de los rizomas de curcuma long I. Cultivada en la Región del Quindío., Revista de Investigaciones Universidad del Quindío, 2008 (18):32-37

(6) Kao, L.; C. Chen y C.J. Chang. Supercritical CO2 extraction of turmerones from turmeric and high-pressure phase equilibrium of $\mathrm{CO} 2$ + turmerones. Journal of Supercritical Fluids. 2007. 43 (2):276-282.

(7)Sharma, R.A.; A.J. Gescher y W.P. Steward. Curcumin: The Story so Far. European Journal of Cancer. 2005. 41 (13): 19551968.

(8) Jang, H.D.; K.S. Chang; Y.S. Huang; C.L. Hsu; S.H. Lee y M.S. Su. Principal phenolic phytochemicals and antioxidant activities of three Chinese medicinal plants. Food Chemistry. 2007. 103 (3): 749-756.

(9)Mandal, V.; Y. Mohan y S. Hemalatha. Microwave assisted extraction of curcumin by sample-solvent dual heating mechanism using Taguchi L9 orthogonal design. Journal of Pharmaceutical and Biochemical Analysis. 2008. 46 (2): $322-$ 327

(10) Péret-Almeida, L.; A.P.F. Cherubino; R.J. Alves; L. Dufossé y M.B.A. Glória. Separation and determination of the physicochemical characteristics of curcumin, demethoxycurcumin and bisdemethoxycurcumin. Food Research International. 2005. 38 (8-9); 1029-1044.

(11) Jiang, H.; B.N. Timmermann \& D.R. Gang. Use of liquid chromatography-electrospray ionization tandem mass spectrometry to identify diarylheptanoids in turmeric (Curcuma longa L.) rhizome. Journal of Chromatography A. 1111 (1): 21-31.

(12)A.O.A.C. Official methods of analysis. 16th Edición. 3a Revision. Association of Official Analytical Chemists. Washington D.C.USA. 1997.

(13) Barrero, M. y R.J. Carreño. Evaluación de los pigmentos de cúrcuma cultivada en Venezuela. Agronomía Tropical. 1999. 49 (4): 491-504. 